MIDPI

MOL2NET, International Conference Series on Multidisciplinary Sciences http://sciforum.net/conference/mol2net-03

sciforum

\title{
QSAR with ETA indices: Insecticidal activity of plant derived compounds against zika virus vector Aedes aegypti
}

Priyanka De (depriyanka8294@gmail.com ) ${ }^{\mathrm{a}}$, Rahul B Aher (rahulba26@gmail.com) ${ }^{\mathrm{a}}$,
and Kunal Roy(kunalroy_in@yahoo.com) ${ }^{\mathrm{a},{ }^{*}}$

${ }^{a}$ Drug Theoretics and Cheminformatics Laboratory,

Department of Pharmaceutical Technology,

Jadavpur University, Kolkata 700 032, India,

Phone: +91 98315 94140; Fax: +91-33-2837-1078;

URL: http://sites.google.com/site/kunalroyindia/

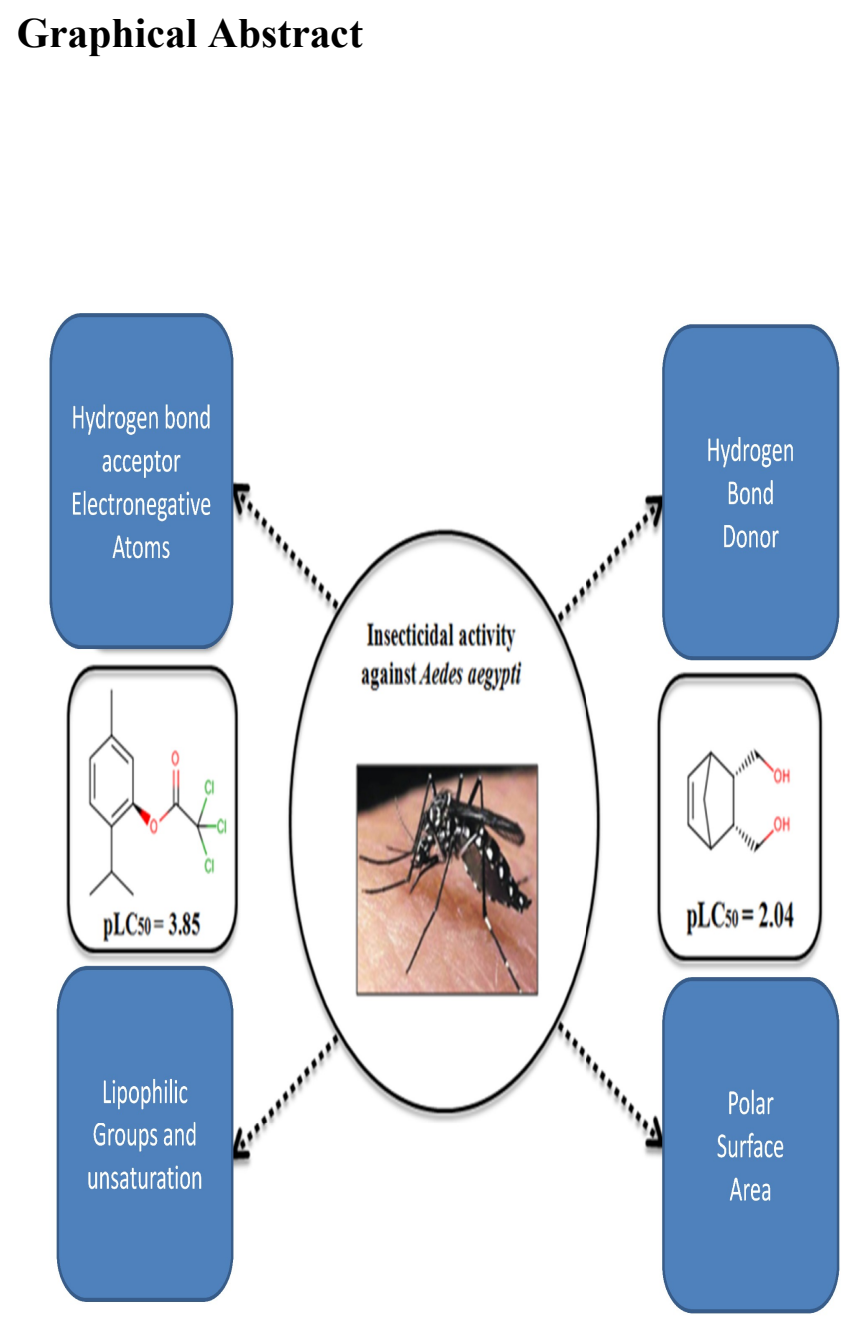

\section{Abstract}

Dengue, zika and chikungunya have severe public health concerns in several countries. Human modification of the natural environment continues to create habitats in which mosquitoes, vectors of a wide variety of human and animal pathogens, thrive which can bring about enormous negative impact on public health if not controlled properly. Quantitative StructureActivity Relationship (QSAR) modeling was applied in this work with the aim to explore features contributing to promising larvicidal and insecticidal property against the vector Aedes aegypti (Diptera:Culicidae). A dataset of 62 plant derived compounds obtained from the previous literatures was used in this present study where Genetic Algorithm (GA) was used for model development employing Double Cross Validation (DCV) tool. Simple topological descriptors like Extended Topochemical Atom (ETA) indices developed by the present authors' group were used for model development. A number of models were generated by the GA method and the descriptors obtained were pooled for Best Subset Selection method (BSS). Further, the best model obtained from BSS was used for Partial Least Square (PLS) regression to 
obtain the final model. The model was validated extensively using different validation metrics to check the robustness and predictivity of the model for regulatory acceptance and enhancing confidence in QSAR predictions. Based on the insights obtained from the PLS model, we can conclude that presence of hydrogen bond acceptor atoms, presence of multiple bonds as well as sufficient lipophilicity and limited polar surface area play crucial roles in regulating the activity of the compounds.

Keywords: QSAR, Zika, Insecticidal activity, Double cross-validation

\section{References}

1. Saavedra, L.M., Romanelli, G.P., Rozo, C.E. and Duchowicz, P.R., 2018. The quantitative structure-insecticidal activity relationships from plant derived compounds against chikungunya and zika Aedes aegypti (Diptera: Culicidae) vector. Science of The Total Environment, 610, pp.937-943.

2. Roy, K. and Ambure, P., 2016. The "double cross-validation" software tool for MLR QSAR model development. Chemometrics and Intelligent Laboratory Systems, 159, pp.108-126.

3. http://teqip.jdvu.ac.in/QSAR Tools/

4. http://dtclab.webs.com/software-tools

5. Roy, K. and Ghosh, G., 2010. Exploring QSARs with Extended Topochemical Atom (ETA) indices for modeling chemical and drug toxicity. Current pharmaceutical design, 16(24), pp.2625-2639. 